\title{
Pandemic preparedness in Taiwan
}

To the Editor - Your June editorial highlights the importance of testing and decisive early control measures for controlling COVID-19. Taiwan has achieved extraordinary success in limiting the impact of SARS-CoV-2 as a result of its early-stage containment policy ${ }^{2}$. Since 9 May, zero confirmed cases have been detected in the country. By 7 June - five months after the World Health Organization declared a global health emergency ${ }^{3}-$ a total of 443 confirmed Taiwanese cases had been recorded and 73,471 people tested $(3,089$ tests per million people), making Taiwan one of the most unaffected countries worldwide, despite its proximity to the virus's source (https://www.cdc.gov.tw/En). However, as SARS-CoV-2 continues to spread worldwide and an outbreak is expected to recur in coming seasons $s^{4}$, advanced preparatory measures continue to be deployed rigorously in Taiwan and the country is actively developing vaccines and medications to combat coronavirus outbreaks.

As a result of the country's pandemic preparedness and early containment policies, the COVID-19 case load in hospitals was brought quickly under control, supplies of protective equipment for healthcare workers were sufficient, and economic disruption was minimized. This meant that production of medical supplies and protective equipment could be scaled up quickly.
In the early months of the pandemic, the government rapidly coordinated enterprises to set up 60 face mask production lines, with production reaching 13 million masks daily by the end of March - a production level sufficient not only for Taiwan but also to aid other countries heavily hit worldwide (https://taiwancanhelp.us).

In addition, a tremendous effort has been made to expand existing SARS-CoV-2 testing capacity in Taiwan to prepare for the next wave of the COVID-19 pandemic, which will surely come. Although some SARS-CoV-2 antigen rapid assay and rapid serological tests for antigen-specific immunoglobulin $\mathrm{M}$ and immunoglobulin $\mathrm{G}$ have been authorized by US Food and Drug Administration Emergency Use Authorization, their effectiveness in confirming disease resistance and curbing the pandemic remain under investigation. The reverse transcription (RT)-PCR test is the only option for early viral infection detection, is more sensitive, and remains the gold standard for COVID-19 diagnosis, which is indispensable for disease control and identifying infected and infectious individuals. However, RT-PCR is a time-consuming and labor-intensive procedure and may expose technicians to unnecessary risk during the sample processing.

Your editorial mentions the problem of existing clinical laboratories being overwhelmed by COVID-19. To solve the increasing loads of COVID-19 testing, companies in Taiwan have taken advantage of automated manufacturing to develop robotic systems aimed at expanding the testing capacity of SARS-CoV-2 PCR assays $^{5}$. A fully automated system was produced by integrating nucleic acid extraction, liquid handling and RT-PCR machines with a pair of six-axis, vertically articulated robot arms (Fig. 1). The throughput is up to 2,016 samples daily, with the added benefits of minimizing contamination risks and reducing burden for laboratory technicians. This robot is expandable and flexible to fit various PCR kits. The test accuracy has been preliminarily validated and now under a final check by Taiwan Centers for Disease $\mathrm{Control}^{6}$. The system is also being submitted for the Emergency Use Authorization recently issued by the Food and Drug Administration?

We consider this machine an efficient tool that may assist countries in need of rapidly expanding testing capacity during this difficult time ${ }^{8}$. The manufacturer is in contact with international humanitarian groups to donate these machines to aid countries in Southeast Asia, Africa, and Central and South America, which remain heavily hit by COVID-19 and still lack sufficient testing capacity.

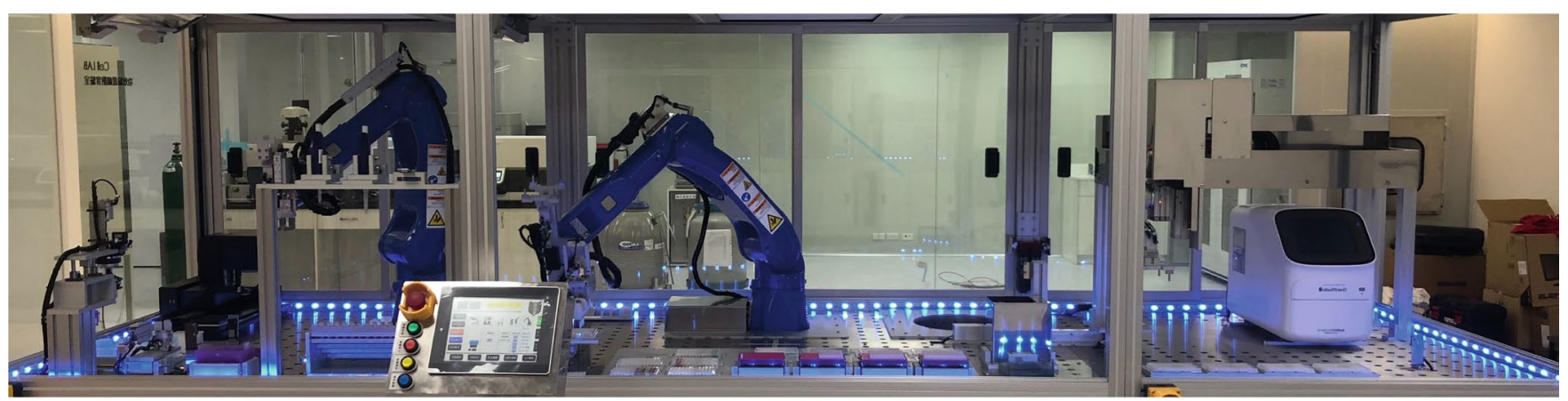

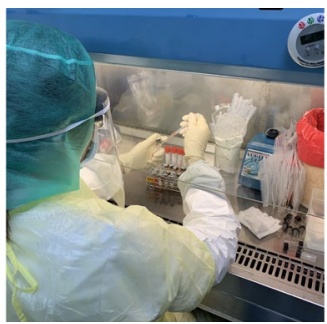

Preprocessing

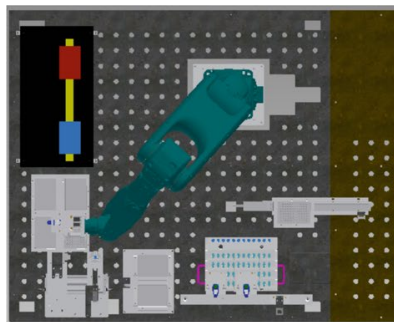

RNA auto-extraction

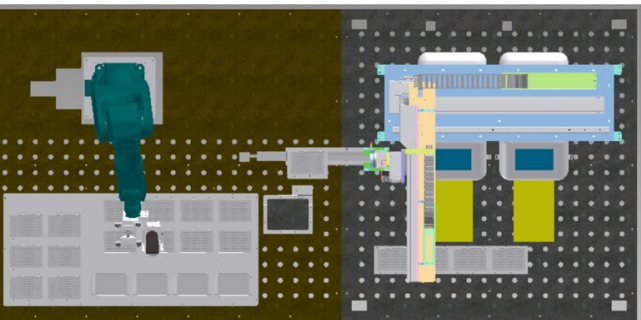

RT-PCR

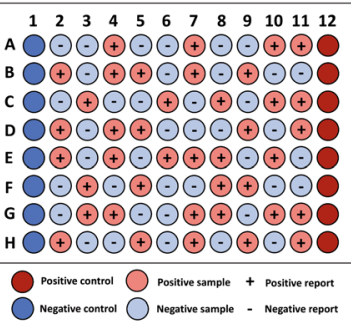

Test validation 
Despite not being a member of the World Health Organization, Taiwan is still doing its best to fight COVID-19 with the world together. We wish our experiences and efforts to be seen and shared to save more lives in the world.

\section{Wei-Kai Wu', Jyh-Ming Liou², Cheng-Chih Hsu (D) ${ }^{3}$, Yung-Hsiang Lin $^{4}$ and Ming-Shiang Wu (D) $2 \otimes$}

${ }^{1}$ Department of Internal Medicine, National Taiwan University Hospital, Bei-Hu Branch, Taipei, Taiwan. ${ }^{2}$ Department of Internal Medicine, National Taiwan
University Hospital, Taipei, Taiwan. ${ }^{3}$ Department of Chemistry, National Taiwan University, Taipei, Taiwan. ${ }^{4}$ Research \& Design Center, TCI Co., Ltd., Taipei, Taiwan.

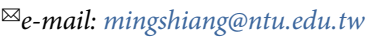

Published online: 23 July 2020

https://doi.org/10.1038/s41587-020-0630-0

References

1. Anonymous. Nat. Biotechnol. 38, 653 (2020)

2. Wang, C. J., Ng, C. Y. \& Brook, R. H. JAMA 323, 1341-1342 (2020).

3. World Health Organization. Statement on the second meeting

of the International Health Regulations (2005) Emergency
Committee regarding the outbreak of novel coronavirus (2019-nCoV). https://www.who.int/news-room/detail/3001-2020-statement-on-the-second-meeting-of-the-intern ational-health-regulations-(2005)-emergency-committeeregarding-the-outbreak-of-novel-coronavirus-(2019-ncov) (2020).

4. Kissler, S. M. et al. Science. 368, 860-868 (2020).

5. O'Meara, S. Nature 577, S1-S3 (2020).

6. TCI Co., Ltd. How has TCI managed "accurate" coronavirus scanning? https://www.tci-bio.com/en-us/official/20200413-qvs96 (2020).

7. Sheridan, C. Nat Biotechnol. 38, 515-518 (2020).

8. Anonymous. Nat. Biomed. Eng. 4, 355-356 (2020).

Y.-H.L. is chairman and president of TCI Co., Ltd.

Check for updates

\section{Early experience with the FDA's Breakthrough Devices program}

To the Editor - The US Congress authorized the Food and Drug Administration (FDA) Breakthrough Devices Program (BDP) in December 2016 in an effort to expedite patient access to innovative devices indicated for the diagnosis and treatment of serious illnesses ${ }^{1}$. In August 2019, the US Centers for Medicare and Medicaid Services (CMS) subsequently finalized changes increasing hospital reimbursement for breakthrough devices to promote clinical adoption ${ }^{2}$. Since program inception, the BDP has grown at a rapid and increasing pace: as of 1 January 2020, the FDA had granted breakthrough designation to 222 devices, representing nearly $200 \%$ program growth over the past year. To shorten device development and review times, the FDA has approved breakthrough devices on the basis of less rigorous premarket evidence with the intention of collecting complementary postmarket data. For example, the FDA has approved breakthrough devices without supporting effectiveness data (for example, an insulin pump) or with substantial safety risks (for example, pneumothorax rates $>30 \%$ ). Nonetheless, breakthrough devices may be commercially available in Europe for years before FDA approval.

As BDP devices now begin to enter the market, patients, physicians and policymakers should understand key features of the BDP and their implications for clinical decision-making. Herein, we detail early FDA experience with the BDP and offer strategies to guide policy and practice moving forward. To strengthen regulation of breakthrough devices, we argue that the FDA should collaborate with regulatory counterparts - particularly those in the European Union - to exploit real-world postmarket experience. Furthermore, the FDA and CMS should coordinate clinical study requirements for breakthrough devices to facilitate Medicare coverage determinations and support clinical decision-making. Such measures may help ensure that the BDP helps deliver safe and effective devices to patients with unmet clinical needs.

\section{BDP program and its provenance}

Ever since Congress granted the FDA authority to regulate medical devices in 1976 , the agency has endeavored to balance the competing priorities of timely access to beneficial new devices and adequate premarket evaluation to protect patient safety. Devices are now regulated under a three-tiered risk classification system: low-risk devices (for example, bandages) are largely exempt from regulatory review; moderate-risk devices (for example, hearing aids) are regulated through either the $510(\mathrm{k})$ or De Novo pathways, which typically require non-clinical evidence for FDA clearance (Supplementary Table 1); and finally, high-risk devices (for example, pacemakers) are primarily evaluated through the Premarket Approval (PMA) pathway, which requires clinical evidence of safety and effectiveness.

For high-risk devices, the time from initiation of pivotal clinical trials - studies that serve as the primary basis for premarket evaluation - to FDA approval generally exceeds 5 years ${ }^{3}$. Both device manufacturers and patient advocacy groups have expressed concern that the FDA review process for high-risk devices is overly cumbersome and impedes access to potentially lifesaving medical devices, including technologies available in other countries ${ }^{4}$. For instance, transcatheter aortic valve replacement, now widely recognized as standard of care for most patients with severe aortic stenosis, was available in 42 countries before gaining US approval ${ }^{5}$. These factors prompted the FDA to introduce the BDP under the $21^{\text {st }}$ Century Cures $\mathrm{Act}^{1}$ and prompted the CMS to revamp reimbursement of devices approved under the program ${ }^{2}$.

The BDP supersedes two previously existing special pathways intended to expedite medical device regulatory review: the Priority Review Program and the Expedited Access Pathway Program (EAP). The Priority Review Program was established in 2013 to place potentially innovative devices at the front of the FDA premarket review queue ${ }^{1}$. However, this proved insufficient at times to expedite review owing to challenges associated with regulating new technical and scientific concepts $^{6}$. In 2015, the FDA established the EAP to overcome such obstacles. The EAP both placed devices at the front of the review queue and offered manufacturers opportunities for premarket collaboration with FDA to facilitate novel regulatory approaches. The BDP now builds upon lessons of the EAP by providing manufacturers additional opportunities to partner with the FDA in shaping device development and regulation (Table 1).

Device manufacturers typically apply for breakthrough designation under the BDP before marketing submission. The FDA determines whether a device merits breakthrough designation on the basis of prespecified criteria on the severity of the target illness and potential clinical 